\title{
Crecimiento en pastoreo rotacional de toretes de razas criollas Romosinuano y Blanco Orejinegro en Colombia
}

\section{Growth in rotational grazing of young bulls from Romosinuano and Blanco Orejinegro creole breeds in Colombia}

\author{
Jaime Quiceno A, ${ }^{1 *}$ Esp, Rodrigo Martinez S, ${ }^{2}$ Ph.D, Henry Mateus E, ${ }^{1}$ Agrólogo, \\ Jaime Gallego G, ${ }^{1}$ MV, Pedro Medina G, 2 M.Sc
}

${ }^{1}$ Corporación Colombiana de Investigación Agropecuaria, CORPOICA, Estación Experimental El Nus, San Roque, Antioquia, Colombia. ${ }^{2}$ Corporación Colombiana de Investigación Agropecuaria, CORPOICA Centro de Investigaciones Tibaitatá, Mosquera, Colombia. *Correspondencia: jquiceno@corpoica.org.co

Recibido: Septiembre de 2010; Aceptado: Agosto de 2011.

\section{RESUMEN}

Objetivo. Evaluar el crecimiento de toretes de las razas Romosinuno (ROMO) y Blanco Orejinegro (BON) en una prueba de comportamiento en pastoreo rotacional. Materiales y métodos. La prueba fue desarrollada en la Estación Experimental El Nus, en la Región Andina Colombiana, donde se evaluaron 20 toretes BON y 16 ROMO provenientes de quince ganaderías comerciales, los cuales fueron mantenidos en un solo grupo durante 221 días en pastoreo rotacional en franjas con periodos cortos de ocupación, de los cuales 56 días fueron en pastoreo no suplementado y 165 días bajo tres diferentes fases de pastoreo suplementado en el potrero. Se realizaron pesajes cada 28 días y se evaluaron variables como: la evolución en peso, la ganancia total y diaria, la diferencia entre pesajes, la relación consumo peso vivo y la tasa de consumo. Resultados. El peso inicial en la raza BON fue $180.4 \pm 36.9$ kilos con 9.6 \pm 1.74 meses de edad y en la raza ROMO fue de $171.8 \pm 32.6$ kilos con $10.1 \pm 3.2$ meses de edad. El incremento general de los individuos entre pesajes fue 0.497 kilos por día para los individuos de raza BON, y 0.366 kilos por día para la raza ROMO. En la prueba de eficiencia la tasa de consumo de suplemento alcanzada fue $64.8 \%$ y $71 \%$ para BON y ROMO respectivamente, equivalentes a una ingestión de materia seca de $0.47 \%$ y $0.53 \%$ con relación al peso vivo. Conclusiones. Este trabajo evidencia un mayor desempeño de los individuos de la raza BON comparados con los animales de la raza ROMO e indica una alta variabilidad en la respuesta a un manejo semi-intensivo en las poblaciones en evaluación.

Palabras clave: Ganancia de peso, mejoramiento genético, pastoreo rotacional (Fuente:CAB). 


\section{ABSTRACT}

Objective. Evaluate the growth performance of young Romosinuano (ROMO) and Blanco Orejinegro (BON) bulls under a rotational grazing system. Materials and methods. The test was performed at the El Nus Experimental Facility, in the Colombian Andean region, where. 20 BON and 16 ROMO young bulls from fifteen commercial livestock farms were evaluated. The animals were kept as one whole group during 221 days in a rotational grazing strip with short occupation periods, out of which they were fed for 56 days through simple grazing and for 165 days under three different grazing phases with food supplements. Weight was taken at 28-day intervals and variables such as: weight evolution, total and daily weight gain, weight differences and the relationship between body weight and intake rate were evaluated. Results. The initial average weight for the BON breed was $180.4 \pm 36.9 \mathrm{~kg}$ for $9.6 \pm 1.74$ months of age, and $171.8 \pm 32.6 \mathrm{~kg}$ for the ROMO breed at an average age of $10.1 \pm 3.2$ months. The general increase for individuals between intervals was $0.497 \mathrm{~kg} / \mathrm{day}$ for the BON breed and $0.366 \mathrm{~kg} /$ day for the ROMO breed. In the efficiency test the food intake rates were $64.8 \%$ and $71 \%$ for BON and ROMO respectively, which represent a dry matter intake of $0.47 \%$ and $0.53 \%$ in relation to live body weight. Conclusions. This work presents a higher performance in BON breed individuals compared to the ROMO breed and indicates a high variability in the response to a semi-intensive grazing system in those evaluated populations.

Key words: Genetic improvement, rotational grazing, weight gain, (Source:CAB).

\section{INTRODUCCIÓN}

Desde el año 2005, la Corporación Colombiana de Investigación Agropecuaria - CORPOICA inició un plan nacional de fomento y multiplicación de las Razas Criollas para realizar investigación participativa integrando el sector público y los ganaderos del país con el propósito de multiplicar, mejorar y fomentar las razas bovinas como la Blanco Orejinegro, Romosinuano, Costeño con Cuernos y Sanmartinero (1). Este plan busca promover la utilización de estos valiosos recursos entre los ganaderos, así como también realizar pruebas de comportamiento y finalmente contribuir al mejoramiento genético de los hatos de ganado criollo del plan de fomento y con la participación de poblaciones comerciales (2).

La selección de toros obedece a varios criterios, entre ellos las medidas de crecimiento, clasificación lineal y valores genéticos para diferentes caracteres. La evaluación de la ganancia posdestete ha sido un método útil para comparar toros bajo condiciones estandarizadas, pero este tipo de evaluaciones tiene varios rasgos que permiten comparaciones validas para determinar el mérito genético asumiendo que las diferencias debidas a condiciones no genéticas predestete no influencia el ordenamiento final para el merito genético del crecimiento posdestete (3).

Las pruebas de comportamiento consisten en la evaluación del potencial del crecimiento después del destete en animales de edades semejantes, mantenidos bajo condiciones ambientales uniformes y pueden utilizarse para la identificación de animales superiores desde el punto de vista genético, Moore et al (4), para su posterior empleo en programas de mejoramiento, aunque el crecimiento después del destete es medianamente heredable en las razas criollas Romosinuano y $\mathrm{BON}(5,6)$.

Los productores de ganado de carne buscan incrementar sus rendimientos mediante la selección para caracteres de crecimiento, pero aunque estas metas de selección han sido importantes, sigue siendo necesario tener una mayor presión de selección para la eficiencia de conversión alimenticia, puesto que se ha determinado que las diferencias entre animales en su capacidad para convertir alimento en ganancia de peso son importantes en la determinación de los ingresos de los productores (4).

Tradicionalmente, una medida de eficiencia del crecimiento es determinada por la eficiencia alimenticia, la cual ha sido establecida como la proporción de alimento, ganancia diaria (conocida por sus siglas en inglés; feed conversión ratio: $\mathrm{FCR}=\mathrm{CMD} / \mathrm{GMD}$ consumo medio diario/ ganancia media diaria) y Feed:Growth, que es la proporción de alimento consumido con respecto a la ganancia de peso, la cual es el reciproco de la eficiencia de ganancia (Growth:Feed), y por esto se incrementa a medida que la eficiencia de la ganancia se disminuye y viceversa. Esta característica esta altamente correlacionada con el crecimiento y se puede confundir con los patrones de madurez de los animales $(7,8)$. Una forma para maximizar la ganancia de los 
sistemas de producción de ganado de carne es minimizar los costos de producción puesto que el suministro alimenticio es uno de los mayores costos de producción (9), por tal motivo, la estimación de la eficiencia alimenticia individual ha sido un aspecto de amplio interés en los últimos años.

El objetivo del presente trabajo fue evaluar la capacidad de crecimiento de toretes de las razas criollas Romosinuano y Blanco Orejinegro bajo pastoreo rotacional, realizando una prueba de eficiencia, como componente de una prueba de comportamiento.

\section{MATERIALES Y MÉTODOS}

Sitio de estudio. La prueba se realizó en la Estación Experimental el Nus de CORPOICA, ubicada en el en el Municipio de San Roque, departamento de Antioquia y localizada geográficamente a $6^{\circ} 29^{\prime}$ de latitud norte y $70^{\circ} 09^{\prime}$ de longitud oeste, $800 \mathrm{msnm}$, temperatura media de $23.2^{\circ} \mathrm{C}, 2.200 \mathrm{~mm} / a$ ño de precipitación y $84 \%$ de humedad relativa.

Animales. Se evaluaron 20 toretes de la raza BON y 16 de la raza ROMO provenientes de quince ganaderías comerciales, seleccionados por los propietarios en las fincas de origen. Luego del arribo de los animales a la Estación, fueron mantenidos en observación en corrales provistos con bebederos y se les realizó examen clínico, pesaje y control de parásitos con un producto a base de ivermectina dosificado de acuerdo al peso. Además, se realizó control de ectoparásitos con producto a base de amitraz, con una repetición a los 120 días. Los toretes fueron vacunados contra fiebre aftosa y carbón sintomático en la época de vacunación general de la región. Además, se realizaron tratamientos contra anaplasma y babesia, con un producto a base de oxitetraciclina, diaminazina y fenildimetil- pirazolona.

Pesaje. El sistema de pesaje fue potrero báscula a intervalos de 28 días, previa recogida y descanso de una hora, para un total de doce pesajes realizados en el mismo horario, ocho en la prueba de comportamiento y cuatro en periodos intermedios de la prueba de eficiencia alimenticia.

Mediciones. Se realizó la medición del perímetro testicular por dos veces, así como las medidas morfométricas de altura a la cruz, altura a la cadera, longitud escápulo humeral tuberosidad isquiática y longitud de la grupa, y las medidas por ultrasonido de área del ojo del lomo (AOL), espesor de la grasa dorsal y profundidad del lomo utilizando un ecógrafo Aloka SSD-500 (MR) con un transductor lineal de $3.5 \mathrm{MHz}$ de 12 centímetros (Aloka Inc., Wallingford, CT, EUA).

Clasificación fenotipica. Los animales fueron valorados y clasificados por un experto de la Asociación Colombiana de Ganado Criollo de acuerdo con el tipo, teniendo en cuenta la apariencia general, los indicadores de adaptación, el desarrollo músculo esquelético y la aptitud reproductiva.

Métodos de campo. Los toretes fueron mantenidos en pastoreo como un solo grupo en 6.4 hectáreas, las cuales correspondieron a 13 franjas en un sistema rotacional. Cada franja fue ocupada por dos a tres días, de acuerdo con el peso total de los animales y la disponibilidad de forraje. Se manejó un registro programado por franja que incluía: fecha de entrada, de salida, días de ocupación y acumulados para mantener periodos de descanso regulados entre 29 y 31 días. El suministro de agua se hizo con bebedero automático de 250 litros y el de sal en un saladero portátil con tapa para que el animal la abra y la cierre, con capacidad para diez kilos y fue utilizada sal mineral del $6.5 \%$ de fósforo.

Fases de la prueba. En la fase primera los animales fueron mantenidos en pastoreo durante 56 días, la segunda, en pastoreo con suministro de suplemento en el potrero en forma masiva y libre, la tercera, con suministro de suplemento en potrero a grupos de seis animales; estas duraron 145 días y la cuarta, o prueba de eficiencia alimenticia en pastoreo con programación y control individual sobre el consumo de suplemento, con una duración de 23 días.

Suplementación. El suplemento alimenticio contenia $80 \%$ maíz molido, $15 \%$ soya estrusionada molida y $5 \%$ melaza, con un valor de $88.5 \%$ de materia de seca y aporte de 119 gramos de proteína y 2.85 megacalorías de energía metabolizable por kilogramo de materia seca. El suplemento siempre se dio en el potrero en comederos plásticos portátiles de $370 \mathrm{~cm}$ de perímetro y capacidad para 250 kilos y se repartió en dos porciones, una en la mañana y otra en la tarde. En la segunda y tercera fase se

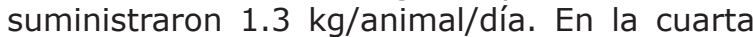
fase se suministró calculado por individuo.

Cuando se ofreció el suplemento en forma masiva y libre, se utilizaron tres y luego seis comederos plásticos. Cada comedero permitió alimentar simultáneamente seis o siete 
animales, los toretes accedieron libremente y el consumo dependió de la habilidad de cada uno para permanecer en el comedero asignado o pasar por los demás. Con el propósito de favorecer el consumo, se pasó a suplementar por grupos de seis animales, para lo cual con bastones e hilo eléctrico en la franja en donde se encontraba el ganado se aislaron áreas para los comederos.

Eficiencia alimenticia. Esta prueba se realizó con el propósito de dar lugar a la capacidad de ganancia de peso de cada animal ante una oferta determinada de suplemento. La ración se calculó de acuerdo con el peso de cada animal, la materia seca del forraje y del suplemento, tendiendo como referencia una ingestión de 3.2\% con relación al peso vivo animal. La cantidad de suplemento programada fue equivalente al $30 \%$ de la ración total, para no exceder los carbohidratos fácilmente fermentables que pudieran producir alteraciones en el rumen. Los aportes de proteína y energía se calcularon utilizando un programador NRC1989 con base en los requerimientos de mantenimiento y una producción deseada de mil gramos animal/día. La conversión alimenticia fue calculada según Nkrumah et al (7).

En la última fase para el suministro de suplemento a cada individuo en la franja, se utilizó el mismo sistema con bastones e hilo eléctrico para aislar áreas con comedero por animal. Además cada día un monitor estuvo encargado de entregar la ración en porciones de 400 gramos, hasta completar lo programado, registrando el tiempo de consumo a cada torete. Durante el periodo de la prueba alimenticia se hicieron cuatro pesajes en periodos cortos para cuantificar la ganancia diaria, establecer la diferencia entre el consumo programado y el realizado o tasa de consumo y calcular un cociente de eficiencia equivalente a la relación consumo ganancia.

Análisis estadístico. Debido a que las fases se manejaron inicialmente como factor de adaptación, solamente se realizó análisis de comparación de medias utilizando el Procedimiento PROC MEANS del programa estadístico SAS (10)

Para las características de peso se realizó un análisis de modelos mixtos con medidas repetidas en el tiempo y utilizando como covarianza la edad del animal y como efecto fijo la raza. El modelo de análisis fue:

$Y_{i j}=\mu+y_{r j}+\beta_{i}\left(x_{i l}+x_{. .}\right)+a_{k}+e_{i j}$
Donde:

$Y_{i j}=$ variable dependiente,

$\mu=$ promedio general,

$\mathrm{y}_{\mathrm{rj}}=$ efecto de raza,

$\beta_{\mathrm{i}}=$ es la pendiente de la covariable edad del animal; $x_{i l}$ es el valor de la covariable para iesima raza en la jesima edad,

$\mathrm{x}=$ es el promedio de la covariable,

$a_{k}=$ efecto aleatorio del kesimo animal y

$\mathrm{e}_{\mathrm{ij}}=$ error experimental.

Los datos fueron analizados utilizando el procedimiento Proc MIXED, utilizando una estructura de (co)varianza autoregresiva 1, que presentó el mejor valor de AIC, se calcularon los componentes de (co)varianza y las soluciones para los efectos fijos y aleatorios, pero en este caso sólo se muestra la significancia de los efectos fijos y para las ecuaciones de crecimiento se realizaron análisis de regresión polinomial, pero las ecuaciones de regresión lineal fueron las que presentaron el mayor ajuste a la variación de la característica. El análisis se realizó utilizando el programa estadístico SAS (10).

\section{RESULTADOS}

La prueba de evaluación productiva incluyó 20 toretes BON procedentes de diez ganaderías colombianas, con peso promedio de $180 \pm 36.9 \mathrm{~kg}$ y edad promedio de $9.6 \pm 1.74$ meses y 16 animales de la raza ROMO, procedentes de otras cinco ganaderías colombianas, con un peso promedio de $171.8 \pm 32.6 \mathrm{~kg}$ y edad promedio de 10.1 \pm 3.2 meses al inicio de la prueba.

Relación suplemento peso vivo. La relación entre la ingestión de suplemento y el peso vivo en las fases segunda de alimentación masiva y tercera por grupo se mantuvo entre $0.65 \%$ y $0.50 \%$ para la raza BON, (Tabla 1 ), mientras que para la raza ROMO estuvieron entre $0.71 \%$ y $0.57 \%$, respectivamente.

Tabla 1. Relación entre la cantidad de suplemento alimenticio y el peso vivo en los diferentes periodos de pesaje de acuerdo con el promedio de peso de los toretes de las razas BON y ROMO.

\begin{tabular}{cccccccc}
\hline RAZA & \multicolumn{2}{c}{$\begin{array}{c}\text { Primera } \\
\text { fase }\end{array}$} & $\begin{array}{c}\text { Segunda } \\
\text { fase }\end{array}$ & $\begin{array}{c}\text { Tercera } \\
\text { fase }\end{array}$ & $\begin{array}{c}\text { Cuarta } \\
\text { fase }\end{array}$ \\
\hline & P 1\% & P 2\% & P 3\% & P 4\% & P 5\% & P 6\% & P 7\% \\
BON & ND & 0.65 & 0.62 & 0.57 & 0.53 & 0.50 & 0.47 \\
ROMO & ND & 0.71 & 0.68 & 0.64 & 0.60 & 0.57 & 0.53 \\
\hline P=Pesaje; & ND $=$ No disponible, periodo sin suplemento. &
\end{tabular}


En la prueba de eficiencia alimenticia la tasa de consumo fue $64.8 \%$ para la raza BON y $71.01 \%$ para la raza ROMO, estos valores correspondieron a un reemplazo en la materia seca de la ración de $19.42 \%$ y $20.89 \%$ respectivamente, los cuales fueron equivalentes a una ingestión de materia seca con respecto al peso vivo de $0.47 \%$ y $0.53 \%$ para cada raza, respectivamente. Aunque todos los animales consumieron suplemento, ningún individuo consumió la cantidad programada.

En la tabla 2, se muestra la cantidad de forraje, proteína cruda (PC) y energía metabolizable (EM) aportadas con el forraje y el suplemento alimenticio suministrado, calculado de acuerdo al consumo promedio por raza, lo que indica que la raza ROMO presentó un menor consumo de forraje y por ende menor suministro proteico y energético; lo mismo ocurrió con el consumo de suplemento.

Tabla 2. Aporte del forraje y el suplemento en la prueba de eficiencia para la raza BON y ROMO en prueba de comportamiento.

\begin{tabular}{cccc}
\hline Raza & EM (Mcal) & PC (g) & $\begin{array}{c}\text { Cantidad } \\
\text { (kg/ms/día) }\end{array}$ \\
\hline BON & 13.55 & 831.38 & 6.52 \\
ROMO & 11.60 & 781.50 & 5.60 \\
& & Suplemento & \\
& EM (Mcal) & PC (g) & $\begin{array}{c}\text { Cantidad } \\
\text { (kg/ms/día) }\end{array}$ \\
BON & 3.96 & 165.46 & 1.39 \\
ROMO & 4.19 & 154.72 & 1.30 \\
& & Eficiencia alimenticia \\
BON & BW075 & GMD & $\mathbf{F C R}$ \\
ROMO & $68.26 \pm$ & $746 \pm 24$ & $14.5 \pm 5.6$ \\
\hline
\end{tabular}

EM: Energía metabolizable; PC: Proteína cruda; BW075: Peso metabólico, GMD: Ganancia media diaria en g., FCR: Feed conversión ratio en kg de materia seca por $\mathrm{kg}$ de ganancia.

En este trabajo se encontraron valores de peso metabólico mayores para la raza BON comparada con la raza ROMO, pero con consumos de alimento y forraje inferiores, por lo que la ganancia media diaria y la eficiencia alimenticia fueron similares y no hubo diferencias significativas entre razas. La eficiencia alimenticia tuvo un valor promedio de $14.5 \mathrm{~kg}$ de materia seca por $\mathrm{kg}$ de ganancia, los valores encontrados para la raza ROMO en ganancia media diaria de peso ( $745 \mathrm{~g}$ de GMD por $\mathrm{kg}$ de ganancia) fueron inferiores que los presentados en la raza BON, para los cuales los menores valores se encontraron cerca a $870 \mathrm{~g}$, que correspondieron a los animales clasificados en los primeros lugares de la prueba.
Evolución de la ganancia de peso en la raza BON. En la figura 1, se muestra la tendencia del peso individual promedio, el cual se incrementó por período de 28 días en $13.9 \mathrm{~kg}$, que corresponde a la pendiente de la curva de crecimiento, la cual tuvo un peso promedio inicial de $180.4 \mathrm{~kg}$ y un peso promedio final de $279.25 \mathrm{~kg}$, con una edad promedio de 17 meses.

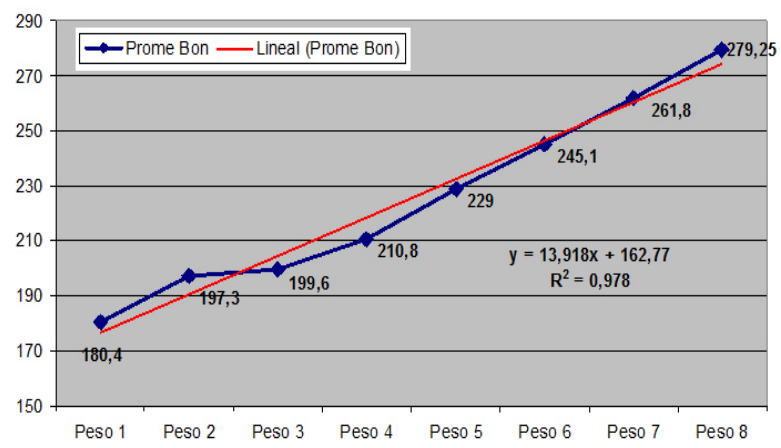

Figura 1. Evolución en peso y tendencia del individuo promedio de la raza BON.

En la tabla 3, se presenta el comportamiento promedio y se describen los valores superiores presentados en el grupo de prueba de la raza BON, teniendo en cuenta las temporadas de lluvia o periodo seco que se presentaron durante el desarrollo. Los pesajes 3 y 4 , coincidieron con el final del periodo de lluvia y fueron los que presentaron las menores ganancias promedio, valores incrementados

Tabla 3. Relación entre épocas, ganancias de peso máxima y superior a 0.7 kg, para la raza BON.

\begin{tabular}{lcccc}
\hline \multirow{2}{*}{ Pesaje } & \multicolumn{4}{c}{ Lluvia } \\
\cline { 2 - 5 } & Sep - Oct & Oct - Nov & Nov - Dic & Dic- Ene \\
\cline { 2 - 5 } & Peso 1 & Peso 2 & Peso 3 & Peso 4 \\
\hline $\begin{array}{l}\text { Peso promedio } \\
\text { Significancia }\end{array}$ & $180 \pm 37$ & $197 \pm 37$ & $200 \pm 39$ & $211 \pm 41$ \\
$\begin{array}{l}\text { Ganancia día } \\
\text { promedio }\end{array}$ & 0.001 & 0.02 & 0.05 & 0.08 \\
$\begin{array}{l}\text { Peso máximo } \\
\text { kg }\end{array}$ & 238 & $607 \pm 392$ & $82 \pm 315$ & $400 \pm 254$ \\
$\begin{array}{l}\text { Ganancia día } \\
\text { Máxima. kg }\end{array}$ & ND & 1.392 & 678 & 1.179 \\
\hline \multicolumn{1}{c}{ Pesaje } & Ene - Feb & Feb - Mar & Mar - Abr & Abril \\
\cline { 2 - 5 } & Peso 5 & Peso 6 & Peso 7 & Peso 8 \\
\hline $\begin{array}{l}\text { Peso promedio } \\
\text { Significancia }\end{array}$ & $229 \pm 46$ & $245 \pm 52$ & $262 \pm 54$ & $279 \pm 56$ \\
$\begin{array}{l}\text { Ganancia día } \\
\text { promedio }\end{array}$ & 0.07 & 0.09 & 0.06 & 0.002 \\
$\begin{array}{l}\text { Peso máximo } \\
\text { kg }\end{array}$ & $648 \pm 300$ & $577 \pm 328$ & $595 \pm 194$ & $621 \pm 178$ \\
\hline Gáxima. kg & 300 & 330 & 346 & 376 \\
\hline
\end{tabular}


para el periodo seco, donde se presenta el mayor valor de ganancia promedio $(648 \pm 300$ $\mathrm{g} /$ animal/día), valor similar al presentado en el pesaje 8 , que correspondió al segundo periodo de lluvias (621 $178 \mathrm{~g} /$ animal/día).

En cuanto a los valores máximos, el mejor comportamiento se dió en el primer período de Iluvia, con valores de 1.392 y $1.179 \mathrm{~g} / \mathrm{animal} /$ día, para los pesajes 2 y 4 , pero igualmente en el primer pesaje del período seco se presentó una ganancia similar ( $1.321 \mathrm{~g} / \mathrm{animal} / \mathrm{día})$ y en el último pesaje del segundo período de Iluvia (1.143 g/animal/día), que correspondió con un individuo que alcanzó $376 \mathrm{~kg}$ a los 17 meses de edad.

Evolución de la ganancia de peso en la raza ROMO. En la figura 2, se presenta una ganancia de peso individual por periodo de 28 días de $10.2 \mathrm{~kg}$, que corresponde a la pendiente de la curva de crecimiento, donde el peso promedio inicial correspondió a 171.8 $\mathrm{kg}$ y un peso promedio final de $245.8 \mathrm{~kg}$, con una edad promedio de 18 meses.

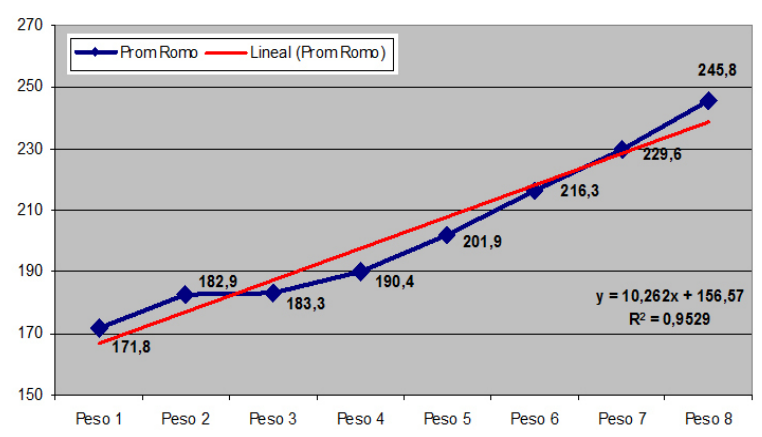

Figura 2. Evolución en peso y tendencia del individuo de la raza ROMO.

En la tabla 4, se muestra la comparación entre medias de los pesos alcanzados por los individuos ROMO. Los pesos del final de la primera temporada de lluvia fueron los menores de todo el período de crecimiento, significativamente inferiores $(p<0.05) \quad(46 \pm 340$ y $330 \pm 247$ para los pesos 3 y 4 en la raza ROMO), igualmente para pesajes posteriores de la época seca, sólo hasta el sexto pesaje, los animales de esta raza lograron sobrepasar una ganancia de $500 \mathrm{~g} /$ animal/día, valor que es inferior al presentado en la raza BON en más de $100 \mathrm{~g}$. Por el contrario, en el último pesaje de la etapa de suministro individual de suplemento, se presentó un valor promedio de ganancia diaria de 578 177 g/animal/ día, valor que sólo es superado en $43 \mathrm{~g}$. por el valor presentado en la raza BON pero sin diferencias significativas $(p>0.05)$.
Tabla 4. Relación entre épocas de lluvia y seca y ganancias de peso promedio y máxima de la raza ROMO.

\begin{tabular}{|c|c|c|c|c|}
\hline \multirow{3}{*}{ Pesaje } & \multicolumn{4}{|c|}{ Lluvia } \\
\hline & \multirow{2}{*}{$\begin{array}{c}\text { Sep - Oct } \\
\text { Peso } 1\end{array}$} & Oct - Nov & Nov - Dic & Dic - Ene \\
\hline & & Peso 2 & Peso 3 & Peso 4 \\
\hline $\begin{array}{l}\text { Peso } \\
\text { promedio }\end{array}$ & $172 \pm 33$ & $183 \pm 35$ & $183 \pm 32$ & $190 \pm 34$ \\
\hline Significancia & 0.001 & 0.001 & 0.05 & 0.08 \\
\hline $\begin{array}{l}\text { Ganancia } \\
\text { promedio }\end{array}$ & & $395 \pm 228$ & $46 \pm 340$ & $330 \pm 247$ \\
\hline $\begin{array}{l}\text { Peso } \\
\text { máximo. kg }\end{array}$ & 233 & 253 & 237 & 253 \\
\hline \multirow[t]{2}{*}{$\begin{array}{l}\text { Ganancia día } \\
\text { Máxima. g }\end{array}$} & & 821 & 571 & 714 \\
\hline & \multicolumn{2}{|c|}{ Seco } & \multicolumn{2}{|c|}{ Lluvia } \\
\hline \multirow[t]{2}{*}{ Pesaje } & Ene - Feb & Feb - Mar & Mar - Abril & Abril \\
\hline & Peso 5 & Peso 6 & Peso 7 & Peso 8 \\
\hline $\begin{array}{l}\text { Peso } \\
\text { promedio }\end{array}$ & $202 \pm 40$ & $216 \pm 44$ & $230 \pm 47$ & $246 \pm 51$ \\
\hline Significancia & 0.09 & 0.06 & 0.06 & 0.002 \\
\hline $\begin{array}{l}\text { Ganancia } \\
\text { promedio }\end{array}$ & $486 \pm 248$ & $511 \pm 342$ & $527 \pm 261$ & $578 \pm 177$ \\
\hline $\begin{array}{l}\text { Peso } \\
\text { máximo. kg }\end{array}$ & 277 & 300.5 & 333 & 353 \\
\hline $\begin{array}{l}\text { Ganancia día } \\
\text { Máxima. g }\end{array}$ & 857 & 1.053 & 1.160 & 964 \\
\hline
\end{tabular}

\section{DISCUSION}

Se evidenciaron diferencias marcadas en el desempeño de los animales en las diferentes fases de la prueba (pastoreo, pastoreo con suplementación y prueba de eficiencia alimenticia), puesto que las ganancias medias diarias se incrementaron en más de $120 \mathrm{~g} /$ animal/día, en términos promedio, este efecto ha sido evidenciado por otros autores como ha sido descrito por Schoeman y Jordaan (11) quienes indicaron que las evaluaciones de la eficiencia en dietas de forrajes y dietas altas en concentrado deberían ser consideradas como dos características independientes en los programas de selección.

En la relación entre la ingestión de suplemento y el peso vivo en las fases segunda de alimentación masiva y tercera por grupo, los rangos diferentes permitieron establecer que los individuos de la raza ROMO mantuvieron un consumo del suplemento alimenticio más alto con respecto al peso.

Los resultados encontrados en la cuarta fase 0 prueba alimenticia sugieren que para mantener un mejor equilibrio en la relación ingestión de suplemento y peso, se deben hacer ajustes de la dieta después de cada pesaje, además, los animales de la raza ROMO presentaron un 
mayor consumo en general, y la diferencia se incrementó, cuando los animales fueron sometidos a la prueba de eficiencia alimenticia.

En las pruebas de comportamiento, diferentes medidas de eficiencia alimenticia han sido propuestas y tradicionalmente, la eficiencia ha sido definida como una proporción de alimento/ ganancia o ganancia/alimento (12). Algunas de estas características de eficiencia de crecimiento conversión y eficiencia de mantenimiento han sido caracterizadas genéticamente (12-14) y se han encontrado valores significativos para el componente genético (15). Por lo que aquellos animales más eficientes consumen menos alimento que el esperado, basado sobre sus requerimientos de mantenimiento y crecimiento de tal forma que los animales más eficientes tienen menores valores para estos índices de conversión (7), en este caso, la raza ROMO presentó menores valores de consumo, pero no se evidenciaron diferencias en la eficiencia alimenticia entre razas.

La incorporación de estas medidas de eficiencia alimenticia dentro de los objetivos de mejora, como puede ser el caso del programa de mejoramiento de estas razas criollas colombianas, puede incrementar el potencial genético de los animales para que tengan menos consumo mientras que sostienen el mismo nivel de producción, puesto que se ha demostrado que los animales más eficientes presentan múltiples beneficios, tales como disminución de consumo de materia seca, menos producción de estiércol y menos emisión de metano $(16,17)$.

En la tendencia del peso individual promedio del individuo BON, es importante resaltar que los pesos tres, cuatro y cinco estuvieron por debajo de la línea de tendencia, debido a la incidencia de hemoparásitos y los efectos del inicio del fenómeno del niño. Al final, en los pesos siete y ocho, el comportamiento del animal promedio superó la línea de tendencia, hecho que puede ser explicado por el efecto del consumo de suplemento individual, dosificado y controlado.

En la raza Romo, con respecto al incremento promedio de peso, se nota un desempeño por debajo de la línea de tendencia, tal como sucedió en la raza BON, pero con la diferencia que el alejamiento de la línea de tendencia fue más amplio, lo cual indicó que la incidencia ambiental y los efectos sanitarios (hemoparásitos) afectaron más fuertemente a los individuos de raza $\mathrm{ROMO}$.

Asímismo, en el pesaje final coincidente con la prueba de eficiencia alimenticia, se nota el incremento en el desempeño de los animales que presentaron valores superiores a la línea de tendencia y dicha variación también fue más amplia que la presentada para la raza BON, lo cual explicó el mejor efecto del suplemento en la prueba de eficiencia con suministro individual, dosificado y controlado para el grupo ROMO.

En la relación entre épocas, ganancias de peso máxima y superior a $0.7 \mathrm{~kg}$ del BON en cuanto a los valores máximos, es de resaltar, que el segundo periodo de pesaje presentó los mayores valores de ganancia diaria, posiblemente debido a un efecto de ganancia compensatoria de algunos animales que se presentaron en la prueba. Mientras que en el ROMO los pesos del final de la primera temporada de lluvia fueron los menores de todo el período de crecimiento, posiblemente debido a efectos ambientales severos, que afectaron en mayor medida, significativamente inferiores.

En cuanto al peso máximo presentado en el primer pesaje, no fue significativamente diferente entre razas, pero este fue cambiando posteriormente, debido a efectos ambientales y en el cuarto pesaje la diferencia fue de $20 \mathrm{~kg}$, tendencia que se mantuvo durante los pesajes sucesivos hasta el sexto pesaje donde la diferencia alcanzó los $30 \mathrm{~kg}$; para el último pesaje esta diferencia retornó a los $23 \mathrm{~kg}(\mathrm{p}<0.05)$.

En esta prueba de comportamiento los animales han sido obtenidos de quince ganaderías localizadas en diferentes regiones de Colombia, animales que fueron sometidos probablemente a diversas condiciones de manejo factor de gran importancia, ya que se ha reportado que el hato de origen $o$ el tratamiento predestete tiene un efecto significativo sobre el peso y ganancia diaria posdestete a 49 y 77 días posdestete, pero también presentó efectos importantes hasta los 189 días posdestete (18). Lo anterior indica que se debe tratar de homogenizar las condiciones de manejo previas al ingreso a la prueba con el fin de eliminar efectos ambientales que puedan interferir en la definición del efecto genético para la selección de los mejores animales en la prueba de comportamiento.

Se puede concluir que las pruebas de comportamiento en peso a potrero, constituyen una herramienta de primera mano para la valoración productiva de los animales a nivel de trópico y los resultados solo sirven para la comparación de los individuos que en ella participen. Es por esto que el periodo de pastoreo no suplementado es necesario para lograr la adaptación de los animales al sistema, a la nueva oferta de forraje, al manejo y a las presiones endémicas de la zona, que en este 
caso afectaron significativamente el desempeño de los animales y pudieron tener un mayor efecto sobre el comportamiento de la raza ROMO, que presentaron un mayor descenso posterior a la presentación de la afección.

En las fases en las cuales se utilizó suplemento, tanto en las de consumo libre como controlado, se demostró que la ingestión de materia seca del suplemento con relación con el peso vivo fue inferior para la raza BON, pero la eficiencia alimenticia no fue significativamente diferente entre razas. Se sugiere en las fases de consumo libre, la utilización de cantidades ajustadas con los pesos obtenidos durante la prueba, para mantener una suplementación más equilibrada.
En conclusión, este trabajo evidenció un mayor desempeño de los individuos de la raza BON comparados con los animales de la raza ROMO e indica una alta variabilidad en la respuesta a un manejo semi-intensivo en estas poblaciones en evaluación.

\section{Agradecimientos}

A los ganaderos participantes en la prueba de comportamiento y al Ministerio de Agricultura y Desarrollo Rural, por el financiamiento del proyecto mediante convenio 2008H710371634, Contrato: 945.

\section{REFERENCIAS}

1. Martínez R, Gallego J, Onofre G, Pérez J. Evaluación de la variabilidad y potencial genético de poblaciones de bovinos criollos colombianos. AGRI 2009; 44(2):45-58.

2. Vásquez R, Gallego J, Pérez J, Jiménez $F$, Onofre G, Polanco N, et al. Multiplicación, mejoramiento y fomento de los bovinos criollos colombianos a través de la creación de núcleos en empresas ganaderas - Plan de fomento de razas bovinas criollas colombianas (PFRBCC). En: X Simposio Iberoamericano Sobre Conservación y Utilización de Recursos Zoogenéticos. Ed. Palmira, Colombia: Universidad Nacional de Colombia; 2009.

3. Baker JF, Vann RC, Neville WE. Evaluations of genotype-environment interactions of beef bulls performance-tested in feedlot or pasture. J Anim Sci 2002; 80:1716-1724.

4. Moore SS, FD Mujibi, E Sherman. Molecular basis for residual feed intake in beef cattle J Anim Sci 2009; 87:41-47.

5. Martinez R, Gallego J, Moreno F. Consanguinity coefficient, phenotypical and genetic characterization of the Colombian Blanco Orejinegro creole breed of cattle. Revista Corpoica 2006; 1:16-24.

6. Martinez R, Pérez J. Estimation of genetic parameters and variance components for growth traits in Romosinuano cattle in the Colombian humid tropics. Gen Mol Res 2007; 6(3):482-491.
7. Nkrumah JD, Basarab JA, Wang Z, Li C, Price MA, Okine EK, et al. Genetic and phenotypic relationships of feed intake and measures of efficiency with growth and carcass merit of beef cattle. J Anim Sci 2007; 85:2711-2720.

8. Hoque MA, M Hosono, T Oikawa, K Suzuki. Relationships with carcass traits of field progeny in Japanese Black cattle Genetic parameters for measures of energetic efficiency of bulls and their J Anim Sci 2009; 87:99-106.

9. Herd RM, Arthur PF. Physiological basis for residual feed intake. J Anim Sci 2009; 87:E64-E71.

10. SAS. Statistical Analysis System Institute Inc. SAS/STAT [Programa estadistico]. Version 9.0. Cary (NC). Users guide statistical analysis system. Institute, Inc. Cory, WC: 2009.

11. Van der Westhuizen RR, Van der Westhuizen J, Schoeman SJ. A genetic analysis of post-weaning feedlot performance and profitability in Bonsmara cattle Genet Mol Res 2009; 25; 8(1):179-96.

12. Herd M, JA Archer, PF Arthur. Reducing the cost of beef production through genetic improvement in residual feed intake: Opportunity and challenges to application. J Anim Sci 2003; 81:9-17. 
13. Arthur PF, JA Archer, DJ Johnson, RM Herd, EC Richardson, PF Parnell. Genetic and phenotypic variance and covariance components for feed intake, feed efficiency and other postweaning traits in Angus cattle. J Anim Sci 2001a; 79:2805-2811.

14. Arthur PF, G Renand, D Krauss. Genetic and phenotypic relationships among different measures of growth and feed efficiency in young Charolais bulls. Livest Prod Sci 2001b; 68:131-139.

15. Crews DH. Genetics of efficient feed utilization and national cattle evaluation: A review. Genet Mol Res 2005; 4:152-165.

16. Nkrumah JD, EK Okine, GW Mathison, $\mathrm{K}$ Schmid, C Li, JA Basarab, et al. Relationships of feedlot feed efficiency, performance, and feeding behaviour with metabolic rate, methane production, and energy partitioning in beef cattle. J Anim Sci 2006; 84:145-153.
17. Hegarty RS, JP Goopy, RM Herd, B McCorkell. Cattle selected for lower residual feed intake have reduced daily methane production. J Anim Sci 2007; 85:1479-1486.

18. Crowley J], M McGee, DA Kenny, DH Crews, Jr, RD Evan, DP Berry. Phenotypic and genetic parameters for different measures of feed efficiency in different breeds of Irish performance-tested beef bulls ] Anim Sci 2010; 88:885-894. 